\title{
Mezcla óptima de producción desde el enfoque gerencial de la contabilidad del throughput: el caso de una pequeña empresa de calzado*
}

doi:10.11144/Javeriana.cc15-37.mopd

\author{
Viviana Karolina Ortiz-T. \\ Ingeniera industrial. Grupo de Investigación en Produc- \\ tividad y Competitividad. Facultad de Ingeniería, Univer- \\ sidad Francisco de Paula Santander, Cúcuta, Colombia. \\ Correo electrónico: vivianakarolinaot@ufps.edu.co
}

\author{
Álvaro Junior Caicedo-R. \\ Ingeniero de producción industrial. Master of Science \\ en ingeniería industrial. Grupo de Investigación en Pro- \\ ductividad y Competitividad. Docente Departamento de \\ Procesos Industriales, Facultad de Ingeniería, Universidad \\ Francisco de Paula Santander, Cúcuta, Colombia. \\ Correo electrónico: alvarojunior450@ufps.edu.co
}

\footnotetext{
* Reporte de caso, como resultado del proyecto Propuesta de un procedimiento para la programación y control de la producción en una pequeña empresa de calzado, realizado en 2012, identificado con el registro 60530 y financiado con recursos propios de los investigadores.
} 
Resumen El propósito de esta investigación fue determinar la mezcla óptima de producción, a partir del enfoque de la contabilidad del throughput, en una pequeña empresa de calzado ubicada en la ciudad de San José de Cúcuta (Colombia), donde se identificaron las restricciones del sistema productivo para el desarrollo de un modelo matemático, siguiendo la teoría de restricciones y la investigación de operaciones, específicamente la técnica de programación lineal. Como resultado, se obtuvieron las cantidades óptimas de fabricación y la secuenciación de producción, que permite obtener mayores utilidades operacionales, para la colección segundo semestre de 2011, dados los recursos productivos disponibles. Además, el estudio permitió identificar la operación crítica del sistema productivo, a partir de lo cual se identificaron escenarios y oportunidades para el aprovechamiento de sus recursos. El modelo matemático diseñado puede implementarse en pequeñas empresas de calzado que presenten las mismas características del caso de estudio.

Palabras clave autor: mezcla óptima de producción; contabilidad de costos; contabilidad del throughput; teoría de restricciones (TOC); programación lineal

Palabras claves descriptor producción teoría económica; teoría de las restricciones (administración); contabilidad de costos; programación lineal.

Códigos JEL: C 6 M 41

\section{Optimal Production Blend from the Management Approach of Throughput Accounting: Case Study of a Small Shoe Company}

\footnotetext{
Abstract The purpose of this research was to determine the optimal output blend from the approach of throughput accounting, in a small shoe company located in San José de Cúcuta City. There the restrictions of the production system for the development of a mathematical model were identified, following the theory
}

of constraints and the operations research, specifically, the linear programming technique. As a result, optimal amounts for manufacturing and production sequencing were obtained, which provides higher operational profits — for the collection of the second half of 2011_, given the available production resources. Also, the study allowed the identification of the critical operation of the production system, from which scenarios and opportunities for the better use of its resources were identified. The mathematical model may be implemented in small shoe companies that have the same characteristics of the case study.

Key words author: optimal production blend; cost accounting; throughput accounting; theory of constraints (TOC); linear programming

Key words plus: production (economic theory); theory of constraints (management); cost accounting; linear programming

\section{Mistura ideal de produção desde o enfoque gerencial da Contabilidade de throughput: o caso de uma pequena empresa de calçado}

Resumo O propósito desta pesquisa foi determinar a mistura ideal de produção, a partir do enfoque da Contabilidade do throughput, em uma pequena empresa de calçado locada na cidade de San José de Cúcuta (Colômbia), onde foram identificadas as restrições do sistema produtivo para o desenvolvimento de um modelo matemático, seguindo a teoria de restrições e a pesquisa de operações, especificamente a técnica de programação linear. Como resultado, obtiveram-se as quantidades ótimas de fabricação e o sequenciamento de produção, que permite obter maiores lucros operacionais, para a coleção segundo semestre de 2011, dados os recursos produtivos disponíveis. Além disso, o estudo permitiu identificar a operação crítica do sistema produtivo, a partir do qual foram identificados cenários e oportunidades para o apro- 
veitamento de seus recursos. O modelo matemático desenhado pode se implementar em pequenas empresas de calçado que apresentarem as mesmas características do caso de estudo

Palavras-chave autor: mistura ideal de produção; contabilidade de custos; contabilidade do throughput; teoria de restrições (TOC); programação linear

Palavras-chave descritor Xteoria das restrições (administração); produção (teoria econômica); contabilidade de custo; programação linear

\section{Introducción}

La mezcla óptima de productos es un tipo de problema de planificación para un solo período, cuya solución proporciona las cantidades óptimas de producción (o mezcla de productos) de un grupo de productos o servicios sujetos a restricciones de capacidad de los recursos disponibles y demanda del mercado (Krajewski, Ritzman \& Malhotra, 2008).

Muchos investigadores reconocen que los problemas de scheduling (programación de la producción) pueden ser resueltos óptimamente utilizando técnicas de programación matemática (Jain \& Meeran, 1999). Un método que permite encontrar las relaciones óptimas que mejor operen un sistema - dado un objetivo específico- es la investigación de operaciones (Prawda, 2004). Un elemento principal de la investigación de operaciones es el modelo matemático (Taha, 2004), aunque hay diversas técnicas para la resolución de estos modelos, Hamdy A. Taha (2004) y Arnold Kaufmann (1978) resaltan la importancia de la técnica de programación lineal. Así mismo, Lee J Krajewski, Larry P. Ritzman y Manoj K. Malhotra
(2008) señalan que la técnica de programación lineal puede utilizarse para encontrar la mejor mezcla de productos.

La importancia de aplicar la investigación de operaciones radica en su fortaleza para modelar problemas complejos y resolver modelos de gran escala (Alvarado, 2011). Otro método es la teoría de restricciones (TOC), que se centra en administrar activamente las restricciones que impiden el progreso de la empresa hacia su meta; ganar dinero hoy y en el futuro (Krajewski, Ritzman \& Malhotra, 2008). Las restricciones del sistema establecen su throughput, que se define como todo el dinero que entra a la empresa menos el dinero que paga a sus proveedores (Corbett, 2001). TOC también es conocida como una filosofía de mejoramiento continuo; las compañías que han adoptado este modo de pensar han obtenido logros sobresalientes ( $\mathrm{Da}$ vies \& Mabin, 2011).

La contabilidad gerencial ya no es capaz de proveer a los gerentes la información necesaria para tomar buenas decisiones. Hay muchas discusiones acerca de cuáles deberían ser las funciones de un sistema de contabilidad gerencial y, en consecuencia, sobre cuál es la información necesaria para tomar decisiones. Por tanto, se necesita información que demuestre que la decisión que se está analizando incrementa la rentabilidad de la empresa. El costeo basado en actividades — ABC — utiliza el análisis de actividades y el costo del producto para verificar si la decisión incrementa la rentabilidad de la empresa. Por otra parte, la contabilidad del throughput utiliza el impacto sobre sus tres medidores (throughput, inversiones y gastos de operación) para responder la misma pregunta 
(Corbett, 2001). Por otro lado, una de las tres medidas básicas para el desempeño de un negocio y que contribuyen con la toma de decisiones es el Throughput Accounting (TA) (Goldratt \& Cox, 2008).

Se han realizado investigaciones con el objetivo de determinar la mezcla óptima de producción. En una microempresa procesadora de filetes de pescado, se implementó un modelo de programación lineal con el fin de determinar las cantidades de fabricación semanal que arrojan la mayor contribución a las utilidades netas de la empresa (Salas, Capachero, Amaya \& Otero, 2007). En un ingenio, se aplicó la técnica de programación lineal para obtener la mezcla óptima de producción para cada tipo de azúcar de exportación, lo que maximizó el margen de contribución para las ventas de exportación; esto facilita la toma de decisiones ante el uso de diferentes recursos limitados en la operación del ingenio (Buenaventura, Moreno, Dussán \& Rivera, 2004). En una planta embotelladora de bebidas gaseosas, Viviana Ortiz \& Álvaro Caicedo (2012) desarrollaron un plan óptimo de producción, a partir de la teoría de restricciones, en conjunto con la técnica de programación lineal.

En pyme del sector muebles de madera se planteó la programación de operaciones, mediante TOC como herramienta para mejorar la producción (Álvarez, Inche \& Salvador, 2004). En una planta de fundición, se aplicó TOC a los procesos de producción, lo cual mejoró el throughput de la restricción identificada respecto a su estado inicial (Abisambra \& Mantilla, 2008). Así mismo, se han realizado estudios para comparar los sistemas de costos y la teo- ría de restricciones, mediante el TA (González, 2006; González \& Escobar, 2008), y modelos de simulación para determinar qué método proporciona mayores beneficios (Ortiz, Nuño de la Parra, Torres \& Báez, 2008).

La estructura productiva de Colombia se caracteriza por la existencia de pequeños establecimientos productivos en los cuales el tamaño de la empresa influye en la capacidad para innovar, competir, exportar y financiarse. Por esto, las mipyme requieren herramientas gerenciales; entre herramientas como los costos ABC, el cuadro de mando integral y la TOC, esta última es la menos conocida (González \& Bermúdez, 2010).

El sector del calzado es muy importante al ser un sector industrial estratégico (ACICAM, 2000; CRCNS, 2010). No obstante, las universidades locales no prestan asistencia científica al sector (CCC, 2007; BRC, 2004). Entre las necesidades del sector, se destaca la de capacitar al empresario de calzado (Estrada, Payán \& Patiño, 2006; Gobernación de Norte de Santander, 2010) acerca de las habilidades técnicas de planificación, programación y control de la producción e inventarios, por medio de herramientas informáticas (Niño, 2011). Otros sectores - como el cerámico - presentan la misma necesidad de capacitación (Caicedo, 2011). Las empresas del calzado que quieran surgir $y$ competir en el entorno actual, deben aumentar su productividad, su competitividad e innovación (Estrada, Payán \& Patiño, 2006), los problemas relacionados con la productividad y la competitividad tienen una participación del $43,7 \%$ y el $34,4 \%$, respectivamente (SENA, 2004). 
El objetivo de esta investigación es determinar la mezcla óptima de producción en una pequeña empresa de calzado, a partir del enfoque de la contabilidad del throughput, lo cual le brindará a la organización una herramienta que le permita conocer las cantidades óptimas a fabricar y la utilización de su sistema productivo. Lo anterior facilita la respuesta de la compañía ante las necesidades de la demanda, al considerar las restricciones del sistema productivo para lograr así maximizar el throughput.

\section{Teoría de restricciones (TOC)}

TOC se centra en el papel que juegan las restricciones en los sistemas, con el fin de mejorar su desempeño hacia la meta. Las restricciones pueden ser de recursos internos, de mercado y de políticas. Los pasos de la teoría de restricciones se pueden observar en la figura 1.

\section{La investigación de operaciones y la técnica de programación lineal}

Las fases principales de la implementación de la investigación de operaciones comprenden la definición del problema, la construcción del modelo, la solución del modelo, la validación del modelo y la implementación de la solución. En la investigación de operaciones, no se tiene una sola técnica general con la que se resuelvan todos los modelos matemáticos que surgen en la práctica; sin embargo, la técnica más importante de investigación de operaciones es la programación lineal (Taha, 2004).

El modelo de programación lineal, como en cualquier modelo de investigación de operaciones, tiene tres componentes básicos: las variables de decisión que se trata de determinar, el objetivo (la meta) que se trata de optimizar y las restricciones que se deben satisfacer (Mathur \& Solow, 1996).

Richard Luebbe y Byron Finch (1992) en su estudio proponen aplicar el procedimiento de la Teoría de restricciones, usando el enfoque de programación lineal (Sipper \& Bulfin, 1998). Así mismo, al hacer una revisión del tema, Jaydeep Balakrishnan y Chun Hung Cheng (2000) concluyeron que: "la programación lineal puede ser vista como una herramienta importante para asegurar que los principios de la Teoría de restricciones sean aplicados correctamente e incrementan el throughput eficientemente".

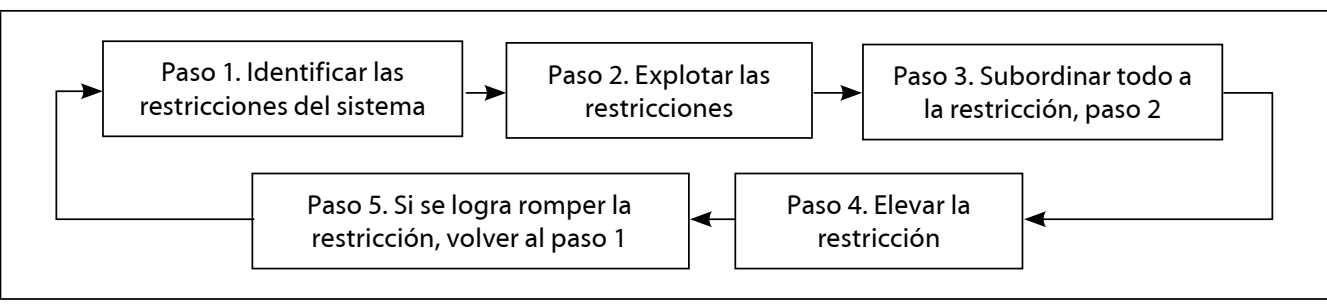

Figura 1. Pasos de la teoría de restricciones Fuente: Daniel Sipper \& Robert L. Bulfin (1998). Planeación y control de la producción. 
Para desarrollar un modelo matemático que permita determinar la mezcla óptima de producción se aplicó la teoría de restricciones (TOC) en conjunto con la investigación de operaciones, específicamente la técnica de programación lineal. Además, los resultados obtenidos fueron confrontados con la contabilidad del throughput y la contabilidad de costos para determinar que método permite obtener mayores utilidades operacionales.

\section{Contabilidad de costos y la contabilidad del throughput}

Según Thomas Corbett (2001), la contabilidad de costos trata de reducir el costo de los productos; esto sucede porque la contabilidad de costos está basada en el supuesto de que entre menor sea el costo de un producto, mayores son las utilidades de la empresa. Como el costo del producto resulta de la utilización que el producto hace de los recursos de la empresa, una forma de reducir el costo de un producto es reduciendo el tiempo de proceso del producto en un recurso. Por otro lado, la contabilidad de costos no es capaz de ofrecer información adecuada, debido a que asume que todos los recursos de la empresa son igualmente importantes. Es como si se tratara de incrementar la resistencia de la cadena fortaleciendo cualquier eslabón, cuando la resistencia de una cadena está determinada por su eslabón más débil; solamente se puede incrementar su resistencia incrementando la resistencia del eslabón más débil. Sin embargo, la contabilidad de costos no percibe a la empresa como un sistema: por esa razón no diferencia entre los recursos de la empresa.
El tiempo disponible de la restricción es limitado. Productos diferentes usan diferentes tiempos de la restricción; el que usa menos tiempo debería tener una prioridad. Así mismo, productos diferentes tienen diferente throughput. Se debe dar prioridad a los productos que tienen un mayor throughput y, al mismo tiempo, dar prioridad a los productos que usan el menor tiempo de la restricción. Para decidir cuál contribuye más a las utilidades de la empresa, se necesita dividir el throughput del producto por el tiempo que usa de la restricción. Además, enfocarse en el throughput obliga a la gerencia a pensar en la empresa como un todo, debido a que solamente se puede incrementar el throughput al optimizar la restricción del sistema y para esto, es necesario tener una visión global. Enfocarse en el throughput ayuda a los gerentes a evitar tratar de optimizar su parte del sistema sin tener en consideración la meta de todo el sistema (Corbett, 2001).

\section{Contabilidad de costos variables y del valor (throughput)}

De acuerdo a lo expuesto por Eric Noreen, Debra Smith y James T. Mackey (1997), el sistema de contabilidad de costos de Eliyahu Goldratt "del Valor" tiene tres bloques principales: throughput, gastos operativos y pasivos. Desde el punto de vista conceptual, el throughput es indistinguible del margen de contribución, throughput es ingresos menos costos totalmente variables; no obstante, en la mayoría de escritos sobre TOC, throughput ha sido definido como ingresos menos materia prima. Y la definición general de margen de contribución es ingresos menos costos variables. Los pasivos (inventarios), término utilizado por Goldratt, se definen 
como todo el dinero que el sistema invierte en la compra de cosas que el propio sistema intenta vender. En la contabilidad del valor, los inventarios, como en el cálculo de los costes variables, consisten únicamente en costos totalmente variables en los que se ha incurrido por parte de artículos en inventario. Los gastos operativos se definen como todo el dinero que el sistema gasta para convertir el inventario en throughput. Son todos los gastos que no han sido deducidos para calcular el throughput.

Desde el punto de vista conceptual, no hay diferencia entre contabilidad del valor y cálculo de costos variables. No obstante, en la práctica hay diferencia significativa: el tratamiento de la mano de obra directa. En la contabilidad del valor, la mano de obra directa no se deduce cuando se calcula el throughput y no es asignada a los inventarios; se incluye como parte de los gastos operativos. En el cálculo de costes variables, el tratamiento convencional considera la mano de obra directa como un coste variable. Es posible argumentar que hoy, en muchas organizaciones, la mano de obra directa es esencialmente un coste fijo, en particular a corto plazo, así la califica Goldratt. En la contabilidad del valor, los resultados financieros pueden ser estimados y comunicados de forma más barata y rápida, y el sistema contable es suficientemente sencillo como para que los resultados financieros puedan ser entendidos fácilmente en la fábrica (Noreen, Smith \& Mackey, 1997).

\section{Caso: pequeña empresa de calzado}

La unidad de estudio es una pequeña empresa de calzado ubicada en la ciudad de San José de Cúcuta, Colombia. Su producción alcanza los 21.282 pares anuales distribuidos entre los 76 productos que allí se elaboran. Enfoca su mercado hacia el calzado para dama, juvenil y niña. Se lanzan dos colecciones por año, en cada colección los modelos cambian sustancialmente, aunque su concepto se mantiene. En cada una de las colecciones, los materiales cambian moderada o totalmente, por lo que el throughput por colección es totalmente variable.

El proceso productivo inicia desde el diseño del producto. En esta etapa se consultan las tendencias del mercado en revistas especializadas, diseños lanzados por la competencia en ferias de calzado de todo el mundo y por observación directa de su mercado objetivo. Posteriormente, siguen las etapas de corte, guarnición (o costura), montado (fijado de la pieza sobre la horma), soleteado (unión de la suela y la pieza montada), y limpieza y empaque. Según la referencia a fabricar, se presentan algunos cambios en las etapas mencionadas anteriormente, incluyendo las operaciones de troquelado y tejido, en la figura 2 se puede observar el flujo productivo de la pequeña empresa de calzado.

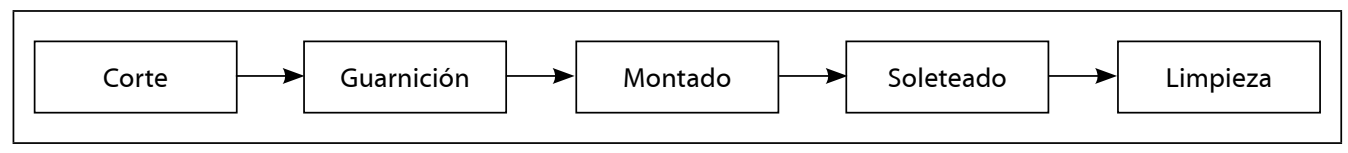

Figura 2. Flujo productivo de la pequeña empresa de calzado Fuente: elaboración propia 
Para abordar el problema de la mezcla óptima de producción, en este estudio de caso se aplicaron los cinco pasos de la teoría de restricciones, en conjunto con la investigación de operaciones, específicamente la técnica de programación lineal.

A continuación, se evidencian los resultados obtenidos al aplicar la teoría de restricciones, junto con la técnica de programación lineal en una pequeña empresa de calzado.

\section{Paso 1. Identificar la restricción del sistema}

Definición de variables de decisión del programa óptimo de producción

Donde:

$\mathrm{C}_{i j}=$ Cantidad de pares de zapatos tipo $i$ a fabricar en el período $j$

i: $\quad$ Índice que identifica el tipo de calzado a fabricar, donde $i=1,2, \ldots, M$

$\mathrm{j}: \quad$ Índice que identifica el período de producción, donde $j=1,2, \ldots, N$

\section{Definición de parámetros constantes del modelo}

Donde:

$\mathrm{U}_{i}=\quad$ Utilidad o throughput por par de zapatos tipo $i$ fabricados, donde: $i=1,2, \ldots$, M

$\mathrm{D}_{i j}=\quad$ Demanda en pares de zapatos tipo $i$ en el período $j$, donde: $i=1,2, \ldots, M y j=$ $1,2, \ldots, N$

$\mathrm{P}_{i j}=\quad$ Cantidad mínima de pedido en pares de zapatos tipo $i$ en el período $j$, donde: $i=$ $1,2, \ldots, M y j=1,2, \ldots, N$
$\mathrm{T}_{i t}=$ Tiempo requerido (en minutos) por par de zapatos tipo $i$, para cada operación tipo $t$ donde: $i=1,2, \ldots, M y t=1$, $2, \ldots, T$

$\mathrm{T}_{t j}=$ Tiempo total disponible (en minutos) para cada operación tipo $t$ en el período j, donde: $t=1,2, \ldots$, Ty $j=1,2, \ldots, N$

$\mathrm{t}$ : Índice que identifica el tipo de operación a realizar, donde $t=1,2, \ldots, \mathrm{T}$

$\mathrm{Cp}_{i j}=$ Cantidad de pares de producto tipo $\mathrm{i}$ que el subcontratista puede procesar en el período $j$, donde: $i=1,2, \ldots, M y j=$ $1,2, \ldots, N$

$\mathrm{TM}_{k i}=$ Consumo de cada material tipo $k$ requerido, en sus respectivas unidades, por par de zapatos tipo $i$, donde: $k=1,2, \ldots$, Ky $i=1,2, \ldots, M$

$\mathrm{k}$ : Índice que identifica el tipo de material requerido, donde $k=1,2, \ldots, K$

$\mathrm{M}_{k j}=$ Cantidad disponible de cada material tipo $k$, en sus respectivos unidades, para la fabricación de calzado en el período j, donde: $k=1,2, \ldots, K y j=1,2, \ldots, N$

\section{Función objetivo}

Para el modelo matemático, el objetivo propuesto es maximizar la utilidad o throughput, la cual viene dada por la expresión:

$$
\operatorname{Max} Z=\sum_{i=1}^{M} \sum_{j=1}^{N} U_{i} * C_{i j}
$$

\section{Identificación de restricciones}

El modelo matemático tiene en cuenta las limitaciones de demanda, cantidad mínima de pedido, capacidad, disponibilidad de materiales y de no negatividad. 


\section{Restricción de demanda}

Los valores de la demanda fueron extraídos de las órdenes de pedido de la feria de calzado realizada en el segundo semestre de 2011.

$C_{i j} \geq D_{i j} \forall i=1,2, \ldots, M ; j=1,2, \ldots, N$

\section{Restricción de cantidad mínima de pedido}

Las cantidades mínimas de pedido fueron establecidas por política de la alta dirección y representan el monto mínimo que debe recibirse de un producto para poder ser enviado a producción.

$$
C_{i j} \geq P_{i j} \forall i=1,2, \ldots, M ; j=1,2, \ldots, N
$$

\section{Restricción de capacidad}

La restricción de capacidad para cada una de las operaciones tipo $t$ se representa en la siguiente ecuación.

$$
\begin{gathered}
\sum_{i=1}^{N} T_{i t} * C_{i j} \leq T_{t j} \\
\forall t=1,2, \ldots, T ; j=1,2, \ldots, N
\end{gathered}
$$

\section{Restricción de capacidad de tejido}

Esta operación es subcontratada; para determinar la capacidad de respuesta del subcontratista, se utilizaron los registros históricos de entrega y los compromisos de cumplimiento.

$$
\sum_{i=1}^{N} C_{i j} \leq C p_{i j}
$$

$$
\forall i=1,2, \ldots, M ; j=1,2, \ldots, N
$$

\section{Restricción de disponibilidad de materiales}

Para determinar la disponibilidad de los materiales se utilizaron los registros de compras, el control de inventarios de materias primas y la experiencia del personal con el fin de calcular la cantidad disponible de los materiales correspondientes y en sus respectivas unidades, para el período seleccionado.

$$
\begin{gathered}
\sum_{i=1}^{N} T M_{k i} * C_{i j} \leq M_{k j} \\
\forall i=1,2, \ldots, M ; j=1,2, \ldots, N
\end{gathered}
$$

De la misma manera, se identificaron las tasas de consumo de los materiales correspondientes en sus respectivas unidades.

\section{Restricción de no negatividad}

Esta restricción hace referencia a que la variable de decisión del modelo deber ser entera y no negativa.

$C i_{j} \in \mathbb{Z}^{+} \forall i=1,2, \ldots, M ; j=1,2, \ldots, N$

Se elaboró un modelo matemático de programación lineal entera que se evidencia en las expresiones enunciadas anteriormente, el cual representa la mezcla óptima de producción para la pequeña empresa de calzado durante el II semestre de 2011. Este modelo permite el aprovechamiento de los recursos productivos, la satisfacción de la demanda y la maximización 
de sus utilidades o throughput, para responder a la problemática identificada en el sector calzado.

\section{Análisis de cargas de trabajo}

De acuerdo con el análisis de cargas de trabajo se identificó el cuello de botella del sistema productivo, paso 1 de la teoría de restricciones. Conociendo las capacidades de producción y los requerimientos de la demanda, se analizaron las restricciones internas que hacen referencia a los recursos productivos de la compañía. Se observa que la operación de guarnición representa el cuello de botella con un porcentaje de utilización de 139,56\%, lo cual refleja una realidad del sector calzado, en el cual el proceso de guarnición es la restricción de todo el sistema de producción (Antolínez, 2004) (cuadro 1). Así mismo, se analizó el porcentaje de utilización de la operación subcontratada; la operación de tejido que aplica a los productos tipo B, cuya capacidad disponible es de 8.840 pares en el período del II semestre de 2011, y la capacidad requerida es de 252 pares en el mismo período, por lo cual la utilización resulta del 2,85\%.

\begin{tabular}{|c|c|c|c|c|c|c|c|}
\hline & & Tiemp & os requ & ueridos por & operación & en minutos & s/par \\
\hline Productos & $\begin{array}{c}\text { Demanda } \\
\text { (pares) }\end{array}$ & Troquelado & Corte & Guarnición & Montado & Soleteado & $\begin{array}{c}\text { Limpieza y } \\
\text { empaque }\end{array}$ \\
\hline G_A & 104 & n.a. & 4,58 & 15,14 & 5,45 & 4,75 & 5,23 \\
\hline G_B & 82 & n.a. & 4,58 & 15,14 & 5,45 & 4,75 & 5,23 \\
\hline G_C & 53 & n.a. & 4,58 & 15,14 & 5,45 & 4,75 & 5,23 \\
\hline G_D & 7 & n.a. & 4,58 & 15,14 & 5,45 & 4,75 & 5,23 \\
\hline G_E & 10 & n.a. & 4,58 & 15,14 & 5,45 & 4,75 & 5,23 \\
\hline G_F & 26 & n.a. & 4,58 & 15,14 & 5,45 & 4,75 & 5,23 \\
\hline J_A & 172 & n.a. & 1,58 & 7,24 & 5,30 & 3,16 & 4,71 \\
\hline J_B & 90 & n.a. & 1,58 & 7,24 & 5,30 & 3,16 & 4,71 \\
\hline J_C & 28 & n.a. & 1,58 & 7,24 & 5,30 & 3,16 & 4,71 \\
\hline J_D & 34 & n.a. & 1,58 & 7,24 & 5,30 & 3,16 & 4,71 \\
\hline J_E & 35 & n.a. & 1,58 & 7,24 & 5,30 & 3,16 & 4,71 \\
\hline J_F & 117 & n.a. & 1,58 & 7,24 & 5,30 & 3,16 & 4,71 \\
\hline J_G & 14 & n.a. & 1,58 & 7,24 & 5,30 & 3,16 & 4,71 \\
\hline J_H & 96 & n.a. & 1,58 & 7,24 & 5,30 & 3,16 & 4,71 \\
\hline B_A & 168 & 3,03 & n.a. & 7,65 & 3,73 & 16,23 & 4,42 \\
\hline B_B & 24 & 3,03 & n.a. & 7,65 & 3,73 & 16,23 & 4,42 \\
\hline B_C & 12 & 3,03 & n.a. & 7,65 & 3,73 & 16,23 & 4,42 \\
\hline B_D & 48 & 3,03 & n.a. & 7,65 & 3,73 & 16,23 & 4,42 \\
\hline $\begin{array}{l}\text { Tiempo total re } \\
\text { operación en co } \\
\text { semestre de } 201\end{array}$ & $\begin{array}{l}\text { querido por } \\
\text { lección II } \\
11 \text { (min) }\end{array}$ & 763,56 & $2.217,44$ & $10.439,92$ & $5.582,66$ & $7.411,06$ & $5.348,76$ \\
\hline $\begin{array}{l}\text { Tiempo total di } \\
\text { operación en co } \\
\text { semestre de } 201\end{array}$ & $\begin{array}{l}\text { sponible por } \\
\text { lección II } \\
11 \text { (min) }\end{array}$ & 7.481 & 7.481 & 7.481 & 7.481 & 7.481 & 7.481 \\
\hline Utilización (\%) & & 10,21 & 29,64 & 139,56 & 74,63 & 99,07 & 71,50 \\
\hline
\end{tabular}

Cuadro 1. Análisis porcentaje de utilización de los recursos para identificar la restricción 
Al determinar el tiempo de producción disponible, se tuvo en cuenta el número de operarios correspondiente, aunque la empresa posee más de un operario por cada operación; a cada uno de ellos se le asigna un trabajo distinto.

Por tanto, se tomó la determinación de hacer el cálculo basado en que solo hay un operario disponible, mientras el resto se dedica a la fabricación de otros productos.

Luego, se determina la cantidad de pares a fabricar con los recursos disponibles actualmente. Para ello, se realiza el montaje y análisis del modelo matemático en el software WinQSB versión 2.0, subprograma Linear and Integer Programming, desarrollado por el doctor YihLong Chang. Los requerimientos de la demanda y la cantidad de pares a fabricar para la colección II semestre de 2011 pueden observarse en el cuadro 2.

La primera letra en mayúscula, en este caso G, J, y B representan cada tipo de producto, y el guion al piso junto con la segunda letra en mayúscula (_A) identifican la combinación correspondiente.

$\mathrm{Al}$ contrastar la cantidad de pares a fabricar con los requerimientos de la demanda, se observa que 8 de los 18 productos presentan pares faltantes por fabricar, lo que arroja como resultado que con los recursos disponibles queda una parte de la demanda insatisfecha.

\section{Paso 2 de la teoría de restricciones, explotar las restricciones}

Se requiere determinar de qué manera se explota la restricción para maximizar su utilidad. El tiempo disponible de la restricción es limitado y los productos diversos usan diferentes tiempos de la restricción, por lo cual se debe dar prioridad a los productos que tienen un mayor throughput, y al mismo tiempo, dar prioridad a los productos que usan el menor tiempo de la restricción. Para decidir cuál contribuye más a las utilidades de la empresa, se necesita dividir el throughput del producto por el tiempo que usa de la restricción (Corbett, 2001). Por otro lado, el principio para maximizar las utilidades totales es el de maximizar la utilidad por unidad de restricción que es cuello de botella (González \& Escobar, 2008).

En el cuadro 3, se calculó el margen de contribución y el margen throughput para cada producto por unidad de restricción (guarnición), a partir de lo cual se determinan las prioridades en la secuencia de producción. 
120 / VOL. 15 / NO. 37 / ENERO-JUNIO 2014

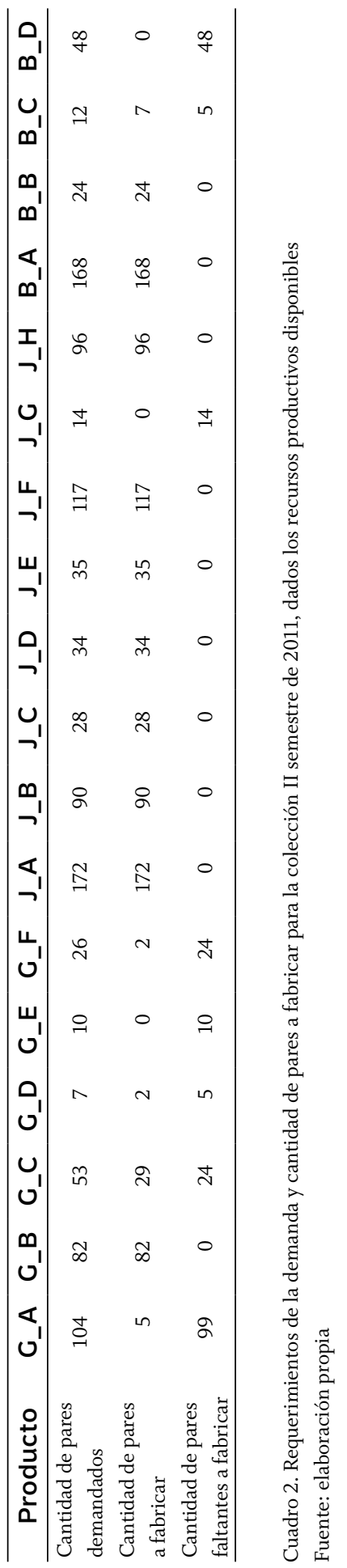




\begin{tabular}{|c|c|c|c|c|c|c|}
\hline Producto & $G_{-} A$ & $G_{-} B$ & G_C & G_D & G_E & $G_{-} F$ \\
\hline Cantidad de pares demandados & 104 & 82 & 53 & 7 & 10 & 26 \\
\hline Precio de venta & $\$ 5.127 .200$ & $\$ 4.042 .600$ & $\$ 2.612 .900$ & $\$ 345.100$ & $\$ 493.000$ & $\$ 1.281 .800$ \\
\hline Costo de materias primas & $\$ 1.267 .448$ & $\$ 999.334$ & $\$ 645.911$ & $\$ 85.309$ & $\$ 121.870$ & $\$ 317.486$ \\
\hline Costo de mano de obra & $\$ 669.656$ & $\$ 527.998$ & $\$ 341.267$ & $\$ 45.073$ & $\$ 64.390$ & $\$ 167.414$ \\
\hline Costos indirectos & $\$ 429.416$ & $\$ 338.578$ & $\$ 218.837$ & $\$ 28.903$ & $\$ 41.290$ & $\$ 107.354$ \\
\hline Contribución marginal & $\$ 2.760 .680$ & $\$ 2.176 .690$ & $\$ 1.406 .885$ & $\$ 185.815$ & $\$ 265.450$ & $\$ 689.546$ \\
\hline $\begin{array}{l}\text { Tiempo de procesamiento en el recursc } \\
\text { cuello de botella ( } \mathrm{min})\end{array}$ & 1574,56 & 1241,48 & 802,42 & 105,98 & 151,40 & 393,64 \\
\hline $\begin{array}{l}\text { Margen de contribución por unidad de } \\
\text { restricción }\end{array}$ & $\$ 1.753$ & $\$ 1.753$ & $\$ 1.753$ & $\$ 1.753$ & $\$ 1.753$ & $\$ 1.752$ \\
\hline Prioridad en producción & 7 & 7 & 7 & 7 & 7 & 8 \\
\hline Valor throughput & $\$ 3.859 .752$ & $\$ 3.043 .266$ & $\$ 1.966 .989$ & $\$ 259.791$ & $\$ 371.130$ & $\$ 964.314$ \\
\hline Margen throughput de la restricción & $\$ 2.451$ & $\$ 2.451$ & $\$ 2.451$ & $\$ 2.451$ & $\$ 2.451$ & $\$ 2.450$ \\
\hline Prioridad en producción & 8 & 8 & 8 & 8 & 8 & 9 \\
\hline Producto & J_A & J_B & J_C & J_D & J_E & J_F \\
\hline Cantidad de pares demandados & 172 & 90 & 28 & 34 & 35 & 117 \\
\hline Precio de venta & $\$ 7.142 .816$ & $\$ 3.737 .520$ & $\$ 1.162 .784$ & $\$ 1.411 .952$ & $\$ 1.453 .480$ & $\$ 4.858 .776$ \\
\hline Costo de materias primas & $\$ 1.689 .040$ & $\$ 883.800$ & $\$ 274.960$ & $\$ 315.418$ & $\$ 344.400$ & $\$ 1.048 .788$ \\
\hline Costo de mano de obra & $\$ 892.508$ & $\$ 467.010$ & $\$ 145.292$ & $\$ 176.426$ & $\$ 181.615$ & $\$ 607.113$ \\
\hline Costos indirectos & $\$ 710.188$ & $\$ 371.610$ & $\$ 115.612$ & $\$ 140.386$ & $\$ 144.515$ & $\$ 483.093$ \\
\hline Contribución marginal & $\$ 3.851 .080$ & $\$ 2.015 .100$ & $\$ 626.920$ & $\$ 779.722$ & $\$ 782.950$ & $\$ 2.719 .782$ \\
\hline $\begin{array}{l}\text { Tiempo de procesamiento en el recurso } \\
\text { cuello de botella ( } \mathrm{min})\end{array}$ & $1.245,28$ & 651,60 & 202,72 & 246,16 & 253,40 & 847,08 \\
\hline $\begin{array}{l}\text { Margen de contribución por unidad de } \\
\text { restricción }\end{array}$ & $\$ 3.093$ & $\$ 3.093$ & $\$ 3.093$ & $\$ 3.168$ & $\$ 3.090$ & $\$ 3.211$ \\
\hline Prioridad en producción & 5 & 5 & 5 & 3 & 6 & 2 \\
\hline Valor throughput & $\$ 5.453 .776$ & $\$ 2.853 .720$ & $\$ 887.824$ & $\$ 1.096 .534$ & $\$ 1.109 .080$ & $\$ 3.809 .988$ \\
\hline Margen throughput de la restricción & $\$ 4.380$ & $\$ 4.380$ & $\$ 4.380$ & $\$ 4.455$ & $\$ 4.377$ & $\$ 4.498$ \\
\hline Prioridad en producción & 5 & 5 & 5 & 3 & 6 & 2 \\
\hline Producto & J_G & J_H & B_A & B_B & B_C & B_D \\
\hline Cantidad de pares demandados & 14 & 96 & 168 & 24 & 12 & 48 \\
\hline Precio de venta & $\$ 581.392$ & $\$ 3.986 .688$ & $\$ 5.807 .424$ & $\$ 829.632$ & $\$ 414.816$ & $\$ 1.659 .264$ \\
\hline Costo de materias primas & $\$ 123.130$ & $\$ 920.544$ & $\$ 2.186 .184$ & $\$ 312.312$ & $\$ 156.156$ & $\$ 624.624$ \\
\hline Costo de mano de obra & $\$ 72.646$ & $\$ 498.144$ & $\$ 1.076 .712$ & $\$ 153.816$ & $\$ 76.908$ & $\$ 307.632$ \\
\hline
\end{tabular}




\begin{tabular}{|c|c|c|c|c|c|c|}
\hline Producto & J_G & J_H & B_A & B_B & B_C & B_D \\
\hline Costos indirectos & $\$ 57.806$ & $\$ 396.384$ & $\$ 693.672$ & $\$ 99.096$ & $\$ 49.548$ & $\$ 198.192$ \\
\hline Contribución marginal & $\$ 327.810$ & $\$ 2.171 .616$ & $\$ 1.850 .856$ & $\$ 264.408$ & $\$ 132.204$ & $\$ 528.816$ \\
\hline $\begin{array}{l}\text { Tiempo de procesamiento en el recurs } \\
\text { cuello de botella ( } \mathrm{min} \text { ) }\end{array}$ & 101,36 & 695,04 & $1.285,20$ & 183,60 & 91,80 & 367,20 \\
\hline $\begin{array}{l}\text { Margen de contribución por unidad de } \\
\text { restricción }\end{array}$ & $\$ 3.234$ & $\$ 3.124$ & $\$ 1.440$ & $\$ 1.440$ & $\$ 1.440$ & $\$ 1.440$ \\
\hline Prioridad en producción & 1 & 4 & 9 & 9 & 9 & 9 \\
\hline Valor throughput & $\$ 458.262$ & $\$ 3.066 .144$ & $\$ 3.621 .240$ & $\$ 517.320$ & $\$ 258.660$ & $\$ 1.034 .640$ \\
\hline Margen throughput de la restricción & $\$ 4.521$ & $\$ 4.411$ & $\$ 2.818$ & $\$ 2.818$ & $\$ 2.818$ & $\$ 2.818$ \\
\hline Prioridad en producción & 1 & 4 & 7 & 7 & 7 & 7 \\
\hline
\end{tabular}

Cuadro 3. Prioridades en la secuencia de producción, de acuerdo al margen de contribución y al margen throughput por producto Fuente: elaboración propia

$\mathrm{Al}$ analizar el margen de contribución tradicional y el throughput, se observa que en ambos casos, algunos productos presentan la misma prioridad en producción. Para solucionar esto, se propone que los productos se ordenen según el tiempo de procesamiento, para dar prioridad a aquellos que requieren menos tiempo del cuello de botella.

Además, la regla de tiempo de procesamiento más breve rinde una solución óptima en el caso de $\mathrm{n} / 1$, para el tiempo promedio de espera y también para el retraso promedio. Esta regla simple es tan potente que se definió como el concepto más importante de todo el ámbito de secuenciación (Chase, Jacobs \& Aquilano, 2009).

En el cuadro 4, se determina la combinación de productos a fabricar para la pequeña empresa de calzado, teniendo en cuenta el margen de contribución tradicional y el margen throughput.

\begin{tabular}{cccccc}
\hline Prioridad & Producto & $\begin{array}{c}\text { Demanda } \\
\text { (pares) }\end{array}$ & $\begin{array}{c}\text { Tiempo } \\
\text { requerido en la } \\
\text { restricción (min) }\end{array}$ & $\begin{array}{c}\text { Tiempo } \\
\text { disponible en } \\
\text { la restricción } \\
\text { (min) }\end{array}$ & $\begin{array}{c}\text { Cantidad de } \\
\text { productos } \\
\text { a fabricar } \\
\text { (pares) }\end{array}$ \\
\hline \multicolumn{5}{c}{ Margen de contribución } \\
\hline 1 & J_G & 14 & 101,36 & 7481 & 14 \\
2 & J_F & 117 & 847,08 & $7.379,64$ & 117 \\
3 & J_D & 34 & 246,16 & $6.532,56$ & 34 \\
4 & J_H & 96 & 695,04 & $6.286,40$ & 96 \\
5 & J_C & 28 & 202,72 & $5.591,36$ & 28 \\
6 & J_B & 90 & 651,60 & $5.388,64$ & 90 \\
7 & J_A & 172 & $1.245,28$ & $4.737,04$ & 172 \\
\hline
\end{tabular}


MEZCLA OPTIMA DE PRODUCCIÓN DESDE LA CONTABILIDAD DEL THROUGHPUT / V. ORTIZ, A. JUNIOR / 123

\begin{tabular}{|c|c|c|c|c|c|}
\hline Prioridad & Producto & $\begin{array}{c}\text { Demanda } \\
\text { (pares) }\end{array}$ & $\begin{array}{l}\text { Tiempo } \\
\text { requerido en la } \\
\text { restricción (min) }\end{array}$ & $\begin{array}{c}\text { Tiempo } \\
\text { disponible en } \\
\text { la restricción } \\
\text { (min) }\end{array}$ & $\begin{array}{l}\text { Cantidad de } \\
\text { productos } \\
\text { a fabricar } \\
\text { (pares) }\end{array}$ \\
\hline \multicolumn{6}{|c|}{ Margen de contribución } \\
\hline 8 & J_E & 35 & 253,40 & $3.491,76$ & 35 \\
\hline 9 & G_D & 7 & 105,98 & $3.238,36$ & 7 \\
\hline 10 & G_E & 10 & 151,40 & $3.132,38$ & 10 \\
\hline 11 & G_C & 53 & 802,42 & $2.980,98$ & 53 \\
\hline 12 & G_B & 82 & $1.241,48$ & $2.178,56$ & 82 \\
\hline 13 & G_A & 104 & $1.574,56$ & 937,08 & 61 \\
\hline 14 & G_F & 26 & 393,64 & 0 & 0 \\
\hline 15 & B_C & 12 & 91,80 & 0 & 0 \\
\hline 16 & B_B & 24 & 183,60 & 0 & 0 \\
\hline 17 & B_D & 48 & 367,20 & 0 & 0 \\
\hline 18 & B_A & 168 & $1.285,20$ & 0 & 0 \\
\hline \multicolumn{6}{|c|}{ Margen throughput } \\
\hline 1 & J_G & 14 & 101,36 & 7481 & 14 \\
\hline 2 & J_F & 117 & 847,08 & $7.379,64$ & 117 \\
\hline 3 & J_D & 34 & 246,16 & $6.532,56$ & 34 \\
\hline 4 & J_H & 96 & 695,04 & $6.286,40$ & 96 \\
\hline 5 & J_C & 28 & 202,72 & $5.591,36$ & 28 \\
\hline 6 & J_B & 90 & 651,60 & $5.388,64$ & 90 \\
\hline 7 & J_A & 172 & $1.245,28$ & $4.737,04$ & 172 \\
\hline 8 & J_E & 35 & 253,40 & $3.491,76$ & 35 \\
\hline 9 & B_C & 12 & 91,80 & $3.238,36$ & 12 \\
\hline 10 & B_B & 24 & 183,60 & $3.146,56$ & 24 \\
\hline 11 & B_D & 48 & 367,20 & $2.962,96$ & 48 \\
\hline 12 & B_A & 168 & $1.285,20$ & $2.595,76$ & 168 \\
\hline 13 & G_D & 7 & 105,98 & $1.310,56$ & 7 \\
\hline 14 & G_E & 10 & 151,40 & $1.204,58$ & 10 \\
\hline 15 & G_C & 53 & 802,42 & $1.053,18$ & 53 \\
\hline 16 & G_B & 82 & $1.241,48$ & 250,76 & 16 \\
\hline 17 & G_A & 104 & $1.574,56$ & 0 & 0 \\
\hline 18 & G_F & 26 & 393,64 & 0 & 0 \\
\hline
\end{tabular}

Cuadro 4. Combinación de productos a fabricar en la pequeña empresa de calzado, según su prioridad en producción Fuente: elaboración propia 
De acuerdo a las secuencias de producción obtenidas en el cuadro 4, se observa que considerando el mayor margen de contribución por unidad de restricción, el sistema de producción alcanza a procesar 13 de los 18 productos, mientras que, considerando el mayor margen del throughput por unidad de restricción, el sistema de producción procesa 16 de los 18 productos. Para elegir la secuencia de producción a utilizar, se identificó la combinación de productos que arroja la mayor utilidad operacional, cuyos resultados se observan en el cuadro 5.

\begin{tabular}{lrlr}
\hline \multicolumn{1}{c}{ Margen de contribución tradicional } & \multicolumn{2}{c}{ Margen throughput } \\
\hline Ingresos & $\$ 34.836 .308$ & Ingresos & $\$ 40.540 .144$ \\
Costo de materias primas & $\$ 8.195 .911$ & Costo de materias primas & $\$ 9.927 .438$ \\
Ingresos - costo de materias primas & $\$ 26.640 .397$ & Ingresos - costo de materias primas & $\$ 30.612 .706$ \\
Gastos de operación & $\$ 7.711 .332$ & Gastos de operación & $\$ 9.024 .772$ \\
\$26.640.397 - gastos de operación & $\$ 18.929 .065$ & $\$ 30.612 .706$ - gastos de operación & $\$ 21.587 .934$ \\
Utilidad operacional & $\$ 18.929 .065$ & Utilidad operacional & $\$ 21.587 .934$ \\
\hline
\end{tabular}

Cuadro 5. Utilidad operacional, según el margen de contribución y el margen throughput Fuente: elaboración propia

De acuerdo a lo expuesto en el cuadro 3, en el cuadro 5 se totalizaron los ingresos, los costos de materias primas y se determinaron los gastos de operación según el margen de contribución y el margen throughput, para calcular la utilidad operacional de cada uno. Como resultado, se obtuvo que la secuencia de producción establecida por el margen throughput genera una mayor utilidad operacional para la empresa, aspecto en el que coincide con estudios similares (González, 2006; González \& Escobar, 2008).

Aun así, esta secuencia no puede aplicarse debido a que los materiales requeridos para la fabricación del calzado tipo J y tipo G presentan demoras en los tiempos de entrega. Por consiguiente, este orden debe modificarse para dar prioridad al calzado tipo B cuyas materias primas llegan primero a planta. La nueva secuencia de producción se puede observar en el cuadro 6.
Al priorizar el calzado tipo B, se observa que aunque cambió la secuencia de producción, la cantidad de productos a fabricar es igual que en el cuadro 4, y la utilidad operacional se mantiene en el valor de $\$ 21.587 .934$, que se obtuvo en el cuadro 5. Teniendo en cuenta los resultados de los cuadros 5 y 6 , se concluye que la secuencia de producción según el margen throughput permite alcanzar una mayor utilidad operacional que la obtenida mediante el margen de contribución.

Luego de maximizar el throughput, se plantea un escenario, en el que se modifica el tiempo de producción disponible para la colección II semestre de 2011. Considerando que la empresa cuenta con personal para ello y para realizar un mejor aprovechamiento de los recursos, se decidió asignar dos operarios para el recurso cuello de botella; en este caso, la operación de guarnición. Los resultados del análisis de cargas de trabajo se muestran en el cuadro 7. 


\begin{tabular}{|c|c|c|c|c|c|}
\hline Prioridad & Producto & $\begin{array}{c}\text { Demanda } \\
\text { (pares) }\end{array}$ & $\begin{array}{l}\text { Tiempo } \\
\text { requerido en la } \\
\text { restricción (min) }\end{array}$ & $\begin{array}{c}\text { Tiempo } \\
\text { disponible en } \\
\text { la restricción } \\
\text { (min) }\end{array}$ & $\begin{array}{c}\text { Cantidad de } \\
\text { productos a } \\
\text { fabricar (pares) }\end{array}$ \\
\hline \multicolumn{6}{|c|}{ Margen Throughput } \\
\hline 1 & B_C & 12 & 91,80 & $7.481,00$ & 12 \\
\hline 2 & B_B & 24 & 183,60 & $7.389,20$ & 24 \\
\hline 3 & B_D & 48 & 367,20 & $7.205,60$ & 48 \\
\hline 4 & B_A & 168 & $1.285,20$ & $6.838,40$ & 168 \\
\hline 5 & J_G & 14 & 101,36 & $5.553,20$ & 14 \\
\hline 6 & J_F & 117 & 847,08 & $5.451,84$ & 117 \\
\hline 7 & J_D & 34 & 246,16 & $4.604,76$ & 34 \\
\hline 8 & J_H & 96 & 695,04 & $4.358,60$ & 96 \\
\hline 9 & J_C & 28 & 202,72 & $3.663,56$ & 28 \\
\hline 10 & J_B & 90 & 651,60 & $3.460,84$ & 90 \\
\hline 11 & J_A & 172 & $1.245,28$ & $2.809,24$ & 172 \\
\hline 12 & J_E & 35 & 253,40 & $1.563,96$ & 35 \\
\hline 13 & G_D & 7 & 105,98 & $1.310,56$ & 7 \\
\hline 14 & G_E & 10 & 151,40 & $1.204,58$ & 10 \\
\hline 15 & G_C & 53 & 802,42 & $1.053,18$ & 53 \\
\hline 16 & G_B & 82 & $1.241,48$ & 250,76 & 16 \\
\hline 17 & G_A & 104 & $1.574,56$ & 0 & 0 \\
\hline 18 & G_F & 26 & 393,64 & 0 & 0 \\
\hline
\end{tabular}

Cuadro 6. Combinación de productos para la pequeña empresa de calzado, para priorizar el calzado tipo B Fuente: elaboración propia

Como resultado, se observa que al asignar dos operarios a la operación de guarnición, esta deja de ser un cuello de botella. Por otro lado, la operación de soleteado presenta un porcentaje de utilización igual al 99,07\%, lo cual indica que este es un recurso de restric- ción de capacidad, aspecto en el que coincide con un estudio realizado por John Wilmer Parra-Llanos (2011). Los requerimientos de la demanda y la mezcla óptima de fabricación al explotar el cuello de botella pueden observarse en el cuadro 8. 


\section{Tiempos requeridos por operación en minutos/par}

\begin{tabular}{|c|c|c|c|c|c|c|c|}
\hline Productos & $\begin{array}{c}\text { Demanda } \\
\text { (pares) }\end{array}$ & Troquelado & Corte & Guarnición & Montado & Soleteado & $\begin{array}{c}\text { Limpieza } \\
y \\
\text { empaque } \\
\end{array}$ \\
\hline G_A & 104 & n.a. & 4,58 & 15,14 & 5,45 & 4,75 & 5,23 \\
\hline G_B & 82 & n.a. & 4,58 & 15,14 & 5,45 & 4,75 & 5,23 \\
\hline G_C & 53 & n.a. & 4,58 & 15,14 & 5,45 & 4,75 & 5,23 \\
\hline G_D & 7 & n.a. & 4,58 & 15,14 & 5,45 & 4,75 & 5,23 \\
\hline G_E & 10 & n.a. & 4,58 & 15,14 & 5,45 & 4,75 & 5,23 \\
\hline G_F & 26 & n.a. & 4,58 & 15,14 & 5,45 & 4,75 & 5,23 \\
\hline J_A & 172 & n.a. & 1,58 & 7,24 & 5,30 & 3,16 & 4,71 \\
\hline J_B & 90 & n.a. & 1,58 & 7,24 & 5,30 & 3,16 & 4,71 \\
\hline $\mathrm{J} \_\mathrm{C}$ & 28 & n.a. & 1,58 & 7,24 & 5,30 & 3,16 & 4,71 \\
\hline J_D & 34 & n.a. & 1,58 & 7,24 & 5,30 & 3,16 & 4,71 \\
\hline J_E & 35 & n.a. & 1,58 & 7,24 & 5,30 & 3,16 & 4,71 \\
\hline J_F & 117 & n.a. & 1,58 & 7,24 & 5,30 & 3,16 & 4,71 \\
\hline J_G & 14 & n.a. & 1,58 & 7,24 & 5,30 & 3,16 & 4,71 \\
\hline J_H & 96 & n.a. & 1,58 & 7,24 & 5,30 & 3,16 & 4,71 \\
\hline B_A & 168 & 3,03 & n.a. & 7,65 & 3,73 & 16,23 & 4,42 \\
\hline B_B & 24 & 3,03 & n.a. & 7,65 & 3,73 & 16,23 & 4,42 \\
\hline B_C & 12 & 3,03 & n.a. & 7,65 & 3,73 & 16,23 & 4,42 \\
\hline B_D & 48 & 3,03 & n.a. & 7,65 & 3,73 & 16,23 & 4,42 \\
\hline \multicolumn{2}{|c|}{$\begin{array}{l}\text { Tiempo total requerido por } \\
\text { operación en colección II } \\
\text { semestre de } 2011 \text { (min) }\end{array}$} & 763,56 & $2.217,44$ & $10.439,92$ & $5.582,66$ & $7.411,06$ & $5.348,76$ \\
\hline \multicolumn{2}{|c|}{$\begin{array}{l}\text { Tiempo total disponible } \\
\text { por operación en colección } \\
\text { II semestre de } 2011 \text { (min) }\end{array}$} & 7.481 & 7.481 & 14.961 & 7.481 & 7.481 & 7.481 \\
\hline \multicolumn{2}{|c|}{ Utilización (\%) } & 10,21 & 29,64 & 69,78 & 74,63 & 99,07 & 71,50 \\
\hline
\end{tabular}

Cuadro 7. Análisis del porcentaje de utilización de los recursos al realizar trabajos en cadena

Fuente: elaboración propia 
MEZCLA OPTIMA DE PRODUCCIÓN DESDE LA CONTABILIDAD DEL THROUGHPUT / V. ORTIZ, A. JUNIOR / 127

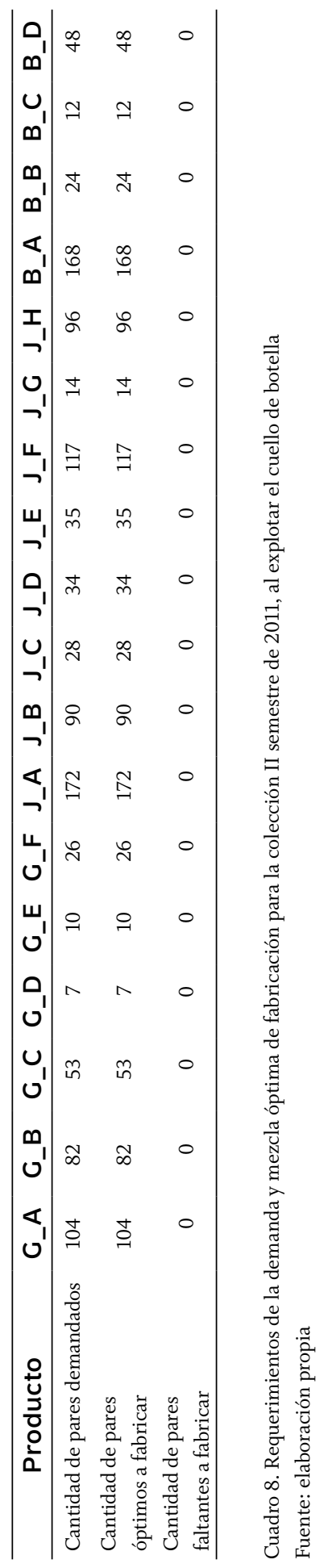


Al explotar el cuello de botella, se observa cómo se les da cumplimiento a todos los requerimientos de la demanda. En este nuevo escenario, se puede implementar la secuencia de producción según el margen throughput que se obtuvo en el cuadro 6.

\section{Paso 3. Subordinar todo lo demás a la restricción}

Para subordinar todo a la restricción, se deben programar los recursos con base en la restricción. Para esto, se sigue el modelo tambor-amortiguador-cuerda, establecido por la teoría de restricciones. El tamaño del amortiguador corresponde a $1 / 4$ o $25 \%$ del tiempo de entrega total real del sistema. Al realizar el cálculo, se tuvo en cuenta que se poseen dos tipos de materias primas: las locales y las nacionales. Los productos que requieren materias primas locales son enviados a producción primero, porque los productos que requieren materias nacionales presentan mayores tiempos de entrega por parte del proveedor. Para los productos que requieren materias primas locales se calcula un primer amortiguador. Así mismo, para los productos que requieren de materias primas nacionales debe calcularse un segundo amortiguador. En el cuadro 9 pueden observarse los resultados obtenidos.

Teniendo en cuenta los tiempos de entrega totales del sistema, se determinó que el tamaño del amortiguador de tiempo es igual a 6.875,86 minutos. Para los productos tipo B, este amortiguador de tiempo es igual a 2.240,27 minutos, lo cual equivale a 62 pares. En el segundo caso, para las 14 referencias restantes, el tamaño del amortiguador de tiempo es igual a 4.635,60 minutos, lo cual equivale a 171 pares.

\section{Paso 4. Elevar la restricción del sistema}

$\mathrm{Al}$ aplicar el paso 2 de la teoría de restricciones se elevó la restricción interna, la cual era la operación de guarnición, lo cual maximizó la utilidad e identificó la operación de soleteado como un recurso de restricción de capacidad.

\section{Paso 5. Volver al paso 1}

Tras haber roto la restricción interna, se inicia un nuevo ciclo de TOC en el que se debe identificar la nueva restricción; en este caso, la restricción a explotar será externa o de mercado, por lo que se recomienda a la empresa establecer estrategias de mercadeo para aumentar las ventas y así aprovechar los recursos productivos disponibles. Además, la empresa iniciaría un proceso de mejora continua, en el que cada vez que se inicie un ciclo de TOC, la empresa debe exigirse para mejorar sus procesos de gestión, que la conduzca al incremento de las utilidades, la productividad y la competitividad.

Además, determinar las cantidades óptimas de fabricación, que permitan el máximo aprovechamiento de los recursos productivos, es indispensable para cualquier empresa que quiera competir y subsistir en un mundo globalizado, en el cual dar soluciones óptimas a los problemas debe ser un asunto vital para toda organización. Por último, el proceso seguido para lograr el objetivo propuesto sirve de referencia para que otras pequeñas empresas con características similares puedan determinar las cantidades óptimas de producción, obtener una mayor utilidad operacional, mientras se satisface la demanda y se da cumplimiento a los compromisos adquiridos con el cliente, lo que genera ventaja competitiva y contribuye al fortalecimiento del sector. 


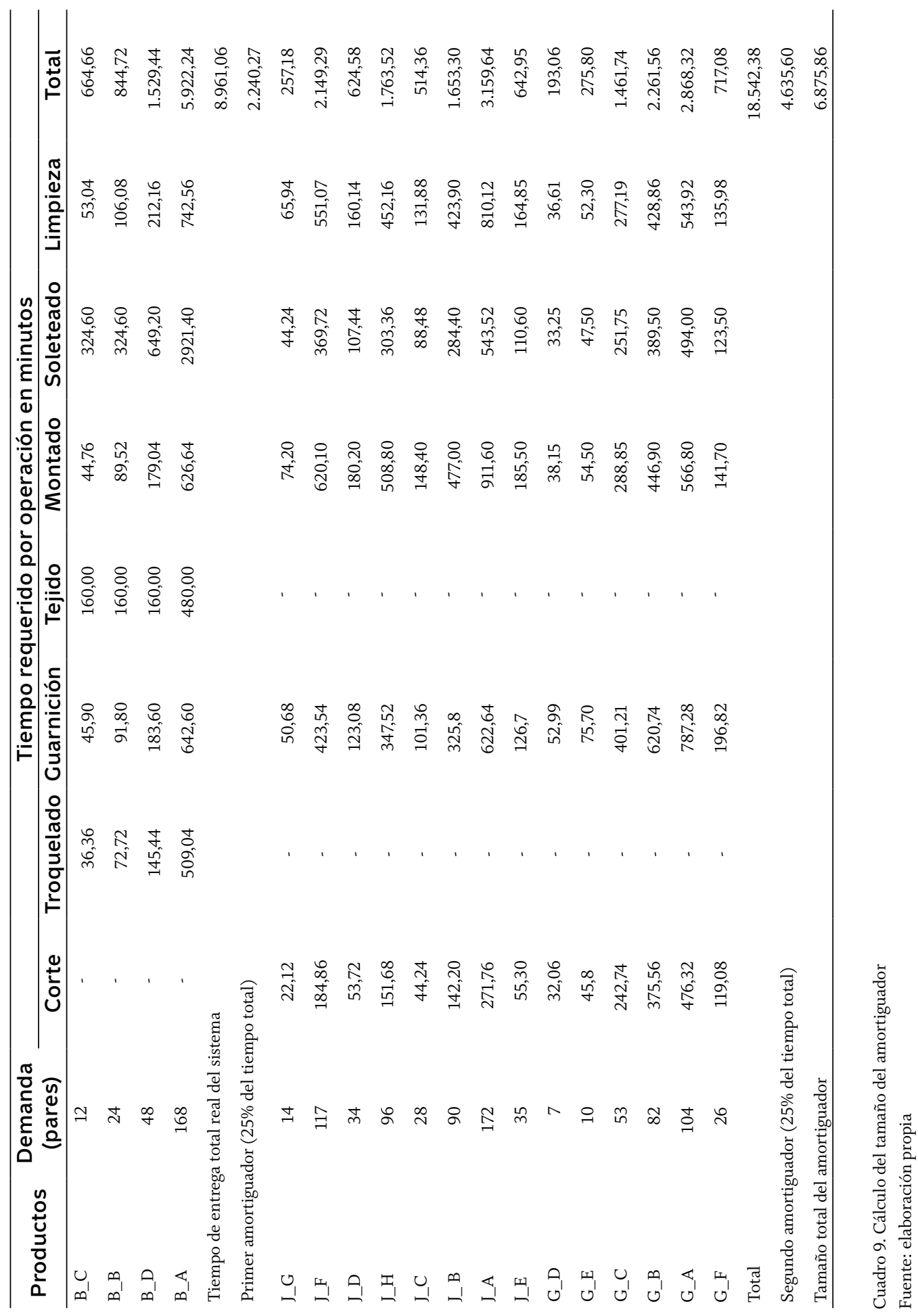




\section{Conclusiones}

Se diseñó un modelo matemático que representa la mezcla óptima de producción de una pequeña empresa de calzado para un período específico; este modelo puede ser utilizado por cualquier pequeña empresa que cuente con las características y operaciones similares a las de la empresa estudiada.

El modelo matemático desarrollado constituye una herramienta con la cual el empresario puede generar una ventaja frente a la competencia y ser más productivo, mientras maximiza sus utilidades o throughput.

Los pasos seguidos para dar solución a la problemática identificada representan un acercamiento novedoso para abordar este tipo de problemas. Además, tener en cuenta las características y funcionamiento del sector permite ofrecer una solución óptima y real para las pequeñas empresas de calzado que deseen aplicarlo.

Se identificó la operación de guarnición como el cuello de botella de la pequeña empresa de calzado, lo cual es acorde con lo establecido por los expertos, con una sobreutilización del 39,56\%. Al explotar la restricción, esta se elevó y la operación de soleteado quedó como un recurso restringido de capacidad, lo que evidencia una capacidad productiva sobrante igual al 0,93 \%. Luego de elevar la restricción y de terminar un ciclo de TOC, se inicia uno nuevo, en el cual la limitante principal de la organización será la demanda, la cual debe explotar para realizar un adecuado aprovechamiento de los recursos productivos.

Al determinar las prioridades de producción, mediante la contabilidad de costos y la contabilidad del throughput (valor) por producto, se identificó que este último obtuvo una mayor utilidad operacional representada en un 14,05\%. Luego de ajustar la secuencia de productos, debido a la disponibilidad de los materiales, la combinación óptima de productos cambió, aunque se mantiene la utilidad operacional obtenida inicialmente mediante el throughput. Sin embargo, para obtener el throughput máximo se deben fabricar todas las cantidades en la secuencia establecida.

\section{Referencias}

Abisambra-Lemus, Abraham José \& MantillaCuadros, Luis Andelfo (2008). Aplicación de la teoría de restricciones (TOC) a los procesos de producción de la planta de fundición de Imusa. Revista Soluciones de Postgrado EIA, Escuela de Ingeniería de Antioquia, 2, 121-133. Disponible en: http:// revistapostgrado.eia.edu.co/Revista $\% 20$ Edici\%F3n\%20N\%BA.2/Soluciones\%20 2\%20art\%209.pdf

Alvarado-Boirivant, Jorge (2011). El análisis post-optimal en programación lineal aplicada a la agricultura. Reflexiones, 90 (1), 161-173, ISSN 1021-1209. Disponible en: http://www.redalyc.org/articulo. oa?id=72918776010

Álvarez, José; Inche, Jorge \& Salvador, Gerardo (2004). Programación de operaciones mediante la teoría de restricciones. Industrial Data, 7 (1), 12-19. Disponible en: http:// www.redalyc.org/pdf/816/81670103.pdf

Cámara de Comercio de Cúcuta, CCC (2007). Visión de Competitividad Regional. Pe- 
riódico El Empresario, 53, 4-5, San José de Cúcuta.

Antolínez, M. (2004). Celdas de manufactura en las pymes. Cómo competir produciendo a bajos costos. Bogotá: Centro Gráfico Salesiano.

Asociación Colombiana de Industriales del Calzado, el Cuero y sus Manufacturas, ACICAM (2000). Plan de mejoramiento de la cadena productiva del calzado, el cuero y sus manufacturas. Bogotá: ACICAM.

Balakrishnan, Jaydeep \& Cheng, Chun Hung (2000). Theory of Constraints and Linear Programming: A Reexamination. International Journal of Production Research, 38 (6), 1459-1463. Disponible en: http://dspace. ucalgary.ca/bitstream/1880/46667/1/Balakrishnan_IJPr_2000a.pdf

Banco de la República de Colombia, BRC (2004). Notas Económicas Regionales. Región Centro Oriente, 3, Bucaramanga. Disponible en: http://www.banrep.gov.co/sites/default/ files/publicaciones/archivos/rco_3.pdf

Buenaventura, Guillermo; Moreno, Andrea; Dussán, Alberto \& Rivera, Orlando (2004). Mezcla óptima de azúcares. Estudios Gerenciales, 92, 141-161. Disponible en: http://estudiosterritoriales.org/articulo. oa?id=21209206

Caicedo, Álvaro Junior (2011). Necesidades de capacitación en habilidades gerenciales para los gerentes de producción del sector de la arcilla del área metropolitana de Cúcuta. Revista Respuestas, 16 (1), 30-37. Disponible en: http://alvarojuniorcaicedo.files. wordpress.com/2011/09/necesidades-decapacitacic3b3n1.pdf
Chang, Yih-Long (2008). WinQSB version 2.0. For Windows 95, 98, ME and 2000. Decision Support Software for MS/OM.

Comisión Regional de Competitividad de Norte de Santander, CRCNS (2010). Avances del Plan Regional de Competitividad a diciembre de 2009. Plan de Acción 2010. Cúcuta: Comisión Regional de Competitividad de Norte de Santander.

Corbett, Thomas (2001). La contabilidad del trúput. El sistema de contabilidad gerencial de TOC. Bogotá: Piénsalo.

Chase, Richard B; Jacobs, F. Robert \& Aquilano, Nicholas J. (2009). Administración de operaciones: producción y cadena de suministros. México: McGraw-Hill.

Davies, John \& Mabin, Vicky (2011). Theory of Constraints. Revista Virtual Pro [en línea] http://www.revistavirtualpro.com/revista/ index.php?ed=2011-09-01\&pag=20

Estrada-Mejía, Sandra; Payán, Andrés Felipe \& Patiño, Heyller (2006). El sector calzado del área metropolitana centro occidente. Rumbo a la productividad y competitividad con ingenio e innovación. Scientia et Technica, 12 (31), 189-194, Disponible en: http://www.redalyc.org/articulo. oa? id=84911639033

Gobernación de Norte de Santander (2010). Análisis comparativo de los sistemas productivos de las mipymes del sector calzado y marroquinería del área metropolitana de Cúcuta. San José de Cúcuta: Gobernación de Norte de Santander.

Goldratt, Eliyahu \& Cox, Jeff (2008). La meta. Buenos Aires: Ediciones Granica S.A. 
González-González, Patricia (2006). Teoría de las restricciones y la mecánica Throughput Accounting. Una aproximación a un modelo gerencial para la toma de decisiones: Caso compañía de Bastones Colombia S.A. Asociación Latinoamericana de Facultades y Escuelas de Contaduría Pública y Administración, ALAFEC [en línea] http://www.alafec.unam.mx/ asam_cuba/ponencias/costos/costos07.doc

González-González, Patricia \& Bermúdez, Tatiana (2010). Fuentes de información, indicadores y herramientas más usadas por gerentes de mipyme en Cali, Colombia. Contaduría y administración [en línea] http://contaduriayadministracionunam.mx/ articulo-2-196-25.html

González-González, Patricia \& Escobar, John Wilmer (2008). Teoría de las restricciones (TOC) y la mecánica del Throughput Accounting (TA). Una aproximación a un modelo gerencial para toma de decisiones: caso compañía de Cementos Andino S.A. Cuadernos de Contabilidad, 7 (24), 209228. Disponible en: http://www.javeriana. edu.co/fcea/cuadernos_contab/vol9_n_24/ vol9_24_7.pdf

Jain, Anant \& Meeran, Sheik (1999). A State of the Art Review of Job Shop Scheduling Techniques. European Journal of Operations Research, 113, 390-434.

Kaufmann, Arnold (1978). Métodos y modelos de la investigación de operaciones. Las matemáticas de la empresa. Tomo 1. México: Compañía Editorial Continental, S.A., CECSA.

Krajewski, Lee J; Ritzman, Larry P. \& Malhotra, Manoj K. (2008). Administración de operaciones. México: Pearson Educación.
Luebbe, Richard \& Finch, Byron (1992). Theory of Constraints and Linear Programming. International Journal of Production Research, 30, 1471-1478.

Mathur, Kamlesh \& Solow, Daniel (1996). Investigación de operaciones. El arte en la toma de decisiones. México: Prentice Hall.

Niño-Rico, Jully Viviana (2011). Plan de capacitación en habilidades gerenciales para los gerentes de producción del sector industrial del calzado, cuero y sus manufacturas en el área metropolitana de Cúcuta. Tesis de pregrado, San José de Cúcuta, Universidad Francisco de Paula Santander.

Noreen, Eric; Smith, Debra \& Mackey, James T. (1997). La teoría de las limitaciones y sus consecuencias para la contabilidad de gestión. Madrid: Ediciones Díaz de Santos.

Ortiz, Fernando; Nuño de la Parra, Pablo; Torres, Raúl \& Báez, Óscar (2008). Comparación del sistema de costos estándar y la teoría de restricciones para el control del flujo de materiales mediante un modelo de simulación. Revista de la Ingeniería Industrial, 2 (1) [en línea] http://academiajournals.com/downloads/Ortiz.pdf

Ortiz, Viviana Karolina \& Caicedo, Álvaro Junior (2012). Plan óptimo de producción en una planta embotelladora de gaseosas. Revista Ingeniería Industrial, 11 (1), 69-82.

Parra-Llanos, John Wilmer (2011). Programación de la producción por medio del modelo de teoría de restricciones, para la empresa de calzado mountain power de la ciudad de Cúcuta. Tesis de pregrado, San José de Cúcuta, Universidad Francisco de Paula Santander. 
Prawda, Juan (2004). Métodos y modelos de investigación de operaciones. Vol. 1. Modelos determinísticos. México: Limusa.

Salas, Rachel; Capachero, Yarley; Amaya, Myleidis \& Otero, Ivonne (2007). Implementación de un modelo de programación lineal para la programación de la producción de filetes de pescado. Caso colombiano. Revista Virtual Pro [en línea] http://www.revistavirtualpro.com/files/ TI08_200709.pdf

Servicio Nacional de Aprendizaje, SENA (2004). Cadena productiva del cuero. Caracterización ocupacional. Actualización. Mesa sectorial el cuero, calzado, y marroquinería. Medellín: SENA. Disponible en: http:// observatorio.sena.edu.co/mesas/01/CADENA\%20CUERO,\%20CALZADO\%20Y\%20 MARROQUINERIA.pdf
Sipper, Daniel \& Bulfin, Robert L. (1998). Planeación y control de la producción. México: McGraw-Hill.

Taha, Hamdy A. (2004). Investigación de operaciones. México: Prentice Hall.

- Fecha de recepción: 26 de julio de 2013

- Fecha de aceptación: 28 de enero de 2014

\section{Para citar este artículo}

Ortiz-T., Viviana Karolina \& Caicedo-R., Álvaro Junior (2014). Mezcla óptima de producción desde el enfoque gerencial de la contabilidad del throughput: el caso de una pequeña empresa de calzado. Cuadernos de Contabilidad, 15 (37), 109-133. 
